\title{
Diseño e implementación de un sistema de medición de respuesta de sensores de gas
}

\section{Design and implementation of a measurement system for gas sensor response}

MUÑOZ-MATA, José Lorenzo†*, ROJAS-GARNICA, Juan Carlos, MUÑOZ-AGUIRRE, Severino y CERVANTES-DE LA ROSA, Juan Pedro

Universidad Tecnológica de Puebla

Benemérita Universidad Autónoma de Puebla

\author{
ID $1^{\mathrm{er}}$ Autor: José Lorenzo, Muñoz-Mata / ORC ID: 0000-0001-7813-5579, CVU CONACYT ID: 177117 \\ ID $1{ }^{\text {er }}$ Coautor: Juan Carlos, Rojas-Garnica / ORC ID: 0000-0002-2261-587X, CVU CONACYT ID: 66417 \\ ID $2^{\text {do }}$ Coautor: Severino, Muñoz-Aguirre / ORC ID: 0000-0002-1822-1464, CVU CONACYT ID: 121589 \\ ID $3^{\text {er }}$ Coautor: Juan Pedro, Cervantes-De La Rosa / ORC ID: 0000-000I-6634-2018
}

DOI: $10.35429 /$ JEA.2019.20.6.26.34

Recibido: 19 de Junio 2019; Aceptado 30 de Septiembre, 2019

\begin{abstract}
Resumen
Los sensores de gas son ampliamente utilizados en dispositivos conocidos como Narices Electrónicas. Los cuales son utilizados para la detección de fugas de gas, calidad del ambiente y alimentos, etc. Sin embargo, es necesario realizar un análisis cuantitativo y cualitativo de dichos sensores a partir de la medición de su respuesta para realizar su caracterización. En el presente trabajo se muestra el diseño e implementación de un sistema de medición de respuesta para sensores de gas. Por lo que se diseña una cámara sellada de acero inoxidable que contenga internamente a los sensores. Por otro lado, se implementa un regulador de temperatura utilizando un controlador PID gobernado por una interfaz desarrollada usando software de instrumentación virtual. Particularmente, este sistema tiene la capacidad de medir la respuesta de sensores de gas, tales como: óxidometálicos y microbalanza de cristal de cuarzo. El desarrollo de este proyecto, presenta una alternativa para la medición de respuesta de sensores a las comerciales, contemplando un menor costo y misma funcionalidad. Se realizaron mediciones a diferentes temperaturas, aplicando muestras de etanol, obteniendo resultados típicos en la respuesta de este tipo de sensores de gas. Por lo tanto, se puede decir que el sistema opera de manera satisfactoria.
\end{abstract}

Nariz Electrónica, Sensores de gas, Control PID

\begin{abstract}
Gas sensors are widely used in devices known as electronic Noses. Which are used for the detection of gas leaks, environmental quality and food, etc. However, it is necessary to perform a quantitative and qualitative analysis of these sensors based on the measurement of their response to obtain their characterization. In the present work is shown the design and implementation of a measurement system for gas sensors response. Therefore, a sealed stainless-steel chamber that internally contains the sensors is designed. On the other hand, a temperature controller is implemented using a PID controller governed by an interface developed using virtual instrumentation software. Particularly, this system has the ability to measure the response of gas sensors, such as: metal-oxide and quartz crystal microbalance. The development of this project, presents an alternative to measure the response of sensors to commercial, contemplating a lower cost and same functionality. Measurements were performed at different temperatures, applying samples of ethanol, obtaining typical results in the response of this type of gas sensors. Therefore, it can be said that the system operates satisfactorily.
\end{abstract}

Electronic Nose, Gas Sensors, PID Control

Citación: MUÑOZ-MATA, José Lorenzo, ROJAS-GARNICA, Juan Carlos, MUÑOZ-AGUIRRE, Severino y CERVANTES-DE LA ROSA, Juan Pedro. Diseño e implementación de un sistema de medición de respuesta de sensores de gas. Revista de Aplicaciones de la Ingeniería. 2019. 6-20: 26-34

\footnotetext{
* Correspondencia del Autor (Correo electrónico: jose.munoz@utpuebla.edu.mx)

$\dagger$ Investigador contribuyendo como primer autor.
} 


\section{Introducción}

Hoy en día los sensores de gas son ampliamente utilizados para diferentes aplicaciones, dado que actualmente la tecnología desarrollada en la nariz electrónica ha avanzado sustancialmente (Nagle, Gutérrez \& Schiffman 1998). Se denomina nariz electrónica a un sistema que detecta e identifica olores y vapores, a través de un arreglo de sensores electroquímicos en conjunto con subsistemas de procesamiento de señales y reconocimiento de patrones (Gardner y Bartlett 1994). Para esto se han utilizado arreglos de sensores los cuales son sensibles a un gran rango de compuestos, así como avanzadas técnicas de reconocimiento de patrones e inteligencia artificial, lo cual permite extraer fácilmente información relevante y confiable. Además, hay una extensa gama de aplicaciones de los sistemas de detección, reconocimiento y discriminación, tales como el monitoreo de la calidad del aire, control de calidad en la industria de alimentos y bebidas, cosmetología, biotecnología, etc. (Gardner y Bartlett 1999).

\section{Sensores de Gas}

Algunos de los sensores de gas utilizados para el desarrollo de narices electrónicas son los de óxido-metal semiconductor y los denominados de microbalanza de cristal de cuarzo QCM. En la Figura 1a, se muestra el sensor de gas semiconductor, el cual está construido con un tubo cerámico de óxido de Aluminio, con una película sensible y un calentador fijo para configurar las condiciones necesarias para el funcionamiento del mismo (Zhengzhou Winsen Electronics Technology Co 2014). En la Figura $1 \mathrm{~b}$ se muestra su circuito interno.

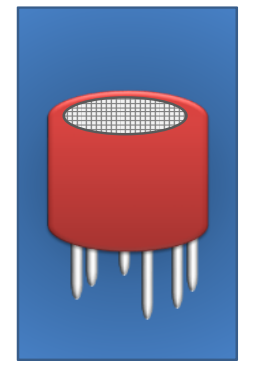

a)

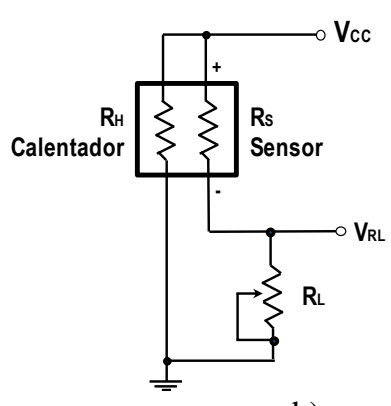

b)
Figura 1 a) Sensor de gas óxido-metálico. b) Circuito Interno

Fuente: Elaboración Propia
El principio de funcionamiento es el siguiente: los sensores de óxido-metal semiconductor aumentan su conductividad al interactuar su superficie con moléculas de gases, dicho cambio de conductividad corresponde a una concentración determinada obteniendo una señal eléctrica con una cierta magnitud, a través de un simple circuito (Ecuación 1).

$\mathrm{R}_{\mathrm{S}}=\left(\frac{\mathrm{V}_{\mathrm{DC}}-\mathrm{V}_{\mathrm{RL}}}{\mathrm{V}_{\mathrm{DC}}}\right) \mathrm{R}_{\mathrm{L}}$

donde $\mathrm{R}_{\mathrm{S}}$ es la resistencia del sensor, $\mathrm{V}_{\mathrm{DC}}$ es el voltaje de alimentación, $V_{R L}$ es el voltaje de salida y $\mathrm{R}_{\mathrm{L}}$ es la resistencia de carga.

Para realizar la medición de respuesta de estos sensores se pueden instrumentar con el uso de un microcontrolador, utilizando el convertidor analógico-digital interno para la adquisición de datos. La visualización y almacenamiento de información se puede crear una interfaz con la computadora.

Por otro lado, los sensores de gas QCM son sensores del tipo piezoeléctrico. Dichos dispositivos pueden medir diferentes variables, en el caso de la nariz electrónica estos son configurados como sensores de cambio de masa (Alassi et al. 2017). En la Figura 2a se muestra el sensor QCM. En el campo científico son de gran importancia debido a la necesidad de detectar, discriminar y reconocer patrones de olores (Gardner y Bartlett 1999). Al aplicar diferentes tipos de películas sensibles en la superficie del electrodo, se podrán obtener diferentes características de cada sensor logrando aplicar criterios más acertados de diferentes gases o vapores (Muñoz-Aguirre et al. 2005).

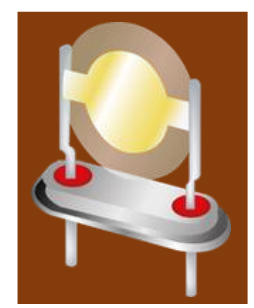

a)

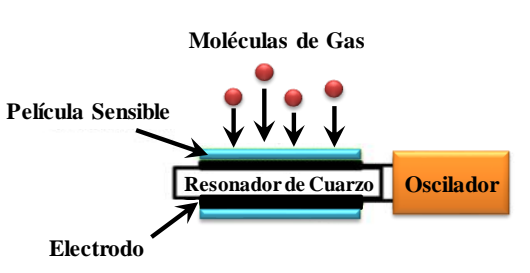

b)
Figura 2 a) Sensor de gas QCM. b) Principio de Operación

Fuente: Elaboración Propia

El principio de funcionamiento se lleva a cabo a través del efecto de adsorción de moléculas en la película sensible (King 1964). 
Al incrementarse la masa, provoca un corrimiento en la frecuencia de resonancia por el efecto de la carga de masa (Arnau 2004), este corrimiento es proporcional a la cantidad de masa que interactúa con la película sensible y se conoce como la respuesta del sensor (MuñozAguirre et al 2005). Sauerbrey muestra dicho corrimiento o cambio de frecuencia en la película sensible en función del cambio de masa por unidad de área en la superficie del electrodo mostrado en la Ecuación 2.

$\Delta \mathrm{f}=-2.3 \times 10^{-6} \cdot \mathrm{F}^{2} \frac{\Delta \mathrm{m}}{\mathrm{A}}$

donde $\Delta \mathrm{f}$ es la variación de la frecuencia $(\mathrm{Hz}),-2.3 \times 10^{-6}$ es una constante determinada por la densidad ( $\rho q)$ y el módulo de cizalla o shear $(\mu \mathrm{q})$ del cuarzo $(\mathrm{m} /(\mathrm{Hz}-\mathrm{g})), \Delta \mathrm{m}$ es la masa adsorbida $(\mathrm{g})$, A es el área del electrodo $\left(\mathrm{cm}^{2}\right)$ y $F$ es la frecuencia de resonancia del cristal $(\mathrm{Hz})$ (Sauerbrey 1959). Por lo tanto, para medir la respuesta de este tipo de sensores es esencial el uso de un frecuencímetro el cual, obtiene el corrimiento de la frecuencia del sensor. Dicha información debe ser enviada a una computadora, para la visualizar y almacenar la información (Muñoz-Mata et al. 2012).

Para realizar el estudio de la respuesta de este tipo de sensores, dado que son altamente sensibles a la temperatura y la humedad es necesario obtener las mediciones en un ambiente controlado para caracterizar los mismos. Por lo que, para controlar la temperatura de exposición del sensor, normalmente estos con introducidos en cámaras de medición, las cuales son sumergidas en tinas o baños térmicos las cuales son de un alto costo y en algunos casos difíciles de adquirir.

El trabajo que se presenta, muestra el diseño e implementación de un sistema de medición para sensores de gas. En este caso particular para sensores de óxido-metálicos semiconductores y sensores de microbalanza de cristal de cuarzo QCM. Se diseñó y manufacturó una cámara de medición de acero inoxidable para poder introducir los sensores. El control de la temperatura se diseñó un control Proporcional-Integral-Derivativo (PID), el cual es manipulado a través de un software de instrumentación virtual, los cuales generan una señal de salida sobre unas celdas Peltier, las cuales fungen como actuadores en el proceso de calentamiento y enfriamiento para el ajuste de la temperatura.
Con la implementación de este sistema se presenta una alternativa de medición a bajo costo para sensores de gas, el cual cubre las necesidades y condiciones necesarias para poder medir la respuesta de estos. Se realizaron mediciones utilizando sensores reales donde se obtuvieron resultados típicos en la respuesta de este tipo de sensores, por lo que se puede decir que el sistema funciona de forma satisfactoria.

\section{Desarrollo Experimental}

Para el desarrollo del sistema se diseñó una cámara de medición de acero inoxidable. Se utilizó un microcontrolador (PIC18F4550Microchip (Microchip 2006)) como interfaz de comunicación con la computadora, para el procesamiento de señales y el envío de la señal de modulación de ancho de pulso (PWM) con el cual se podrá regular el actuador de temperatura. La medición de la temperatura se realizó con el uso del sensor digital DS18B20 (Dallas Semiconductor 2018). El desarrollo de software se con LabVIEW. En la Figura 3, se muestra el diagrama de bloques del sistema implementado.

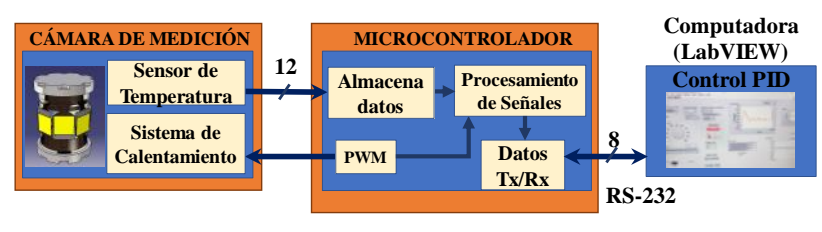

Figura 3 Diagrama a bloques del sistema implementado Fuente: Elaboración Propia

\section{Cámara de Medición}

La cámara de medición fue diseñada con el software de diseño asistido por computadora CATIA (Torrecilla 2013). Dicho dispositivo está compuesto de tres partes principales, un cuerpo central y dos tapas con cierre hermético. La primera parte o cuerpo central está compuesta de un tubo de acero inoxidable con ocho caras planas que sirven de soporte a los actuadores. Por el hecho de que el tubo sea de acero inoxidable y que el espesor en las caras planas fluctúe entre 1 y $3 \mathrm{~mm}$ se favorece la transferencia de calor hacia el interior de la cámara. Los extremos se encuentran roscados para la colocación de tapas. La segunda parte está conformada por las tapas, las cuales están hechas de aluminio, las cuales también cuentan con el mismo roscado del cuerpo central que, con ayuda de un sello de poliuretano, garantiza la hermeticidad de la cámara en el momento de inyectar los gases a evaluar.

MUÑOZ-MATA, José Lorenzo, ROJAS-GARNICA, Juan Carlos, MUÑOZ-AGUIRRE, Severino y CERVANTES-DE LA ROSA, Juan Pedro. Diseño e implementación de un sistema de medición de respuesta de sensores de gas. Revista de Aplicaciones de la Ingeniería. 2019. 
En una de las tapas se encuentran los conectores para los sensores térmicos y de gas, así como los conectores neumáticos para la inyección del gas y para la purga de este. En la Figura 4 se pueden observar las vistas isométricas del diseño de la cámara de medición, donde se aprecian los ensambles de los componentes previamente mencionados.
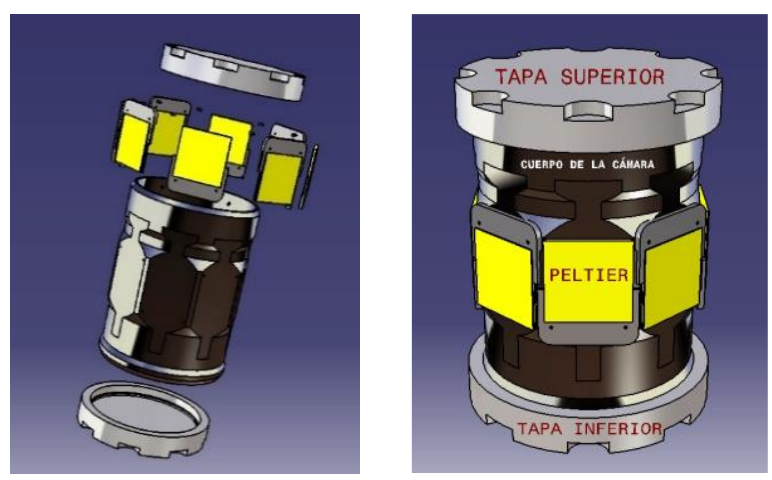

Figura 4 Vistas isométricas de la cámara de medición Fuente: Elaboración Propia

Adicionalmente, se colocó al interior de la cámara, el sensor de temperatura. Dicho sensor servirá como retroalimentación para cerrar el lazo de control del sistema.

\section{Interfaz de comunicación}

Se diseñó un firmware para realizar una interfaz y establecer la comunicación entre la cámara de medición y una computadora utilizando un microcontrolador (PIC18F4550-Microchip), como se muestra en la Figura 3. La interfaz recibirá la información proveniente del sensor de temperatura, la cual será el lazo de retroalimentación de la ley de control y en función de esta señal, la computadora enviará la señal proporcional al ancho de pulso (PWM) que el microcontrolador genera para la regulación de la temperatura deseada establecida por la entrada o setpoint del sistema de control. La comunicación bidireccional realizada por el microcontrolador será a través de la comunicación RS-232.

\section{Desarrollo de Software}

El desarrollo de software fue realizado con LabVIEW (Lajara y Pelegrí 2011), el cual contiene el algoritmo donde se implementó una ley de control utilizando un controlador PID, el cual se encarga de reducir el error producido en la salida con respecto del valor deseado (entrada), como se muestra en la Ecuación 3.

$$
u(t)=K_{p} e(t)+\frac{K_{p}}{\tau_{i}} \int_{0}^{t} e(t)+K_{p} \tau_{d} \frac{d e(t)}{d t},
$$

donde $u(t)$ es la salida del sistema, $e(t)$ es la señal de error, $K_{p}$ es la ganancia proporcional, $\tau_{\mathrm{i}}$ es la constante de tiempo integral, $\tau_{\mathrm{d}}$ es la constante de tiempo derivativa.

Tomando en cuenta que el sistema tiene un comportamiento en tiempo continuo, es necesario discretizar la señal de control para poder aplicar un algoritmo a través de la computadora. Por lo tanto, para poder digitalizar el control PID se utilizó la transformada Z. De esta manera poder determinar el comportamiento del sistema en tiempo discreto (Ogata 1996). De manera que el control PID en tiempo discreto se muestra en la Ecuación 4.

$$
u(z)=e(z) K_{p}\left[1+\frac{T}{\tau_{i}\left(1-z^{-1}\right)}+\tau_{d} \frac{\left(1-z^{-1}\right)}{T}\right]
$$

donde $\mathrm{K}_{\mathrm{p}}$, es la ganancia proporcional, $\tau_{\mathrm{i}}$, y $\tau_{\mathrm{d}}$ son constantes de tiempo expuestas en la Ecuación 3 y T es el tiempo de muestreo. En este caso el tiempo de muestreo es de $300 \mathrm{~ms}$. El algoritmo se desarrolló con el uso del software de instrumentación virtual LabVIEW. El diagrama de flujo del algoritmo de control implementado se muestra en la Figura 5.

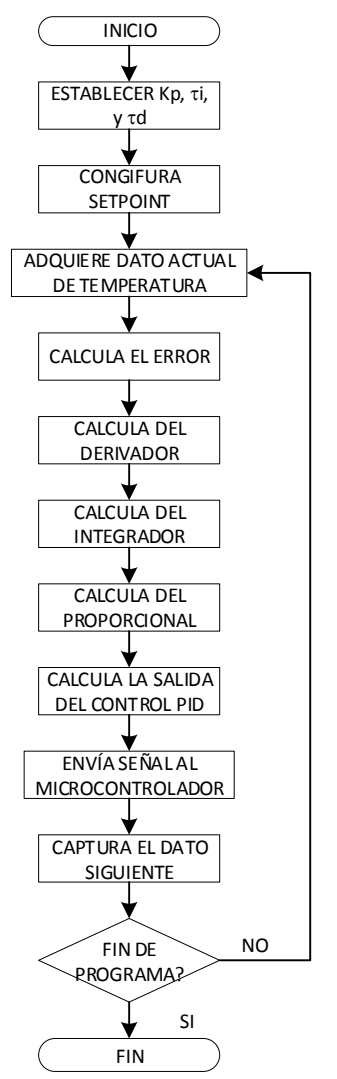

Figura 5 Diagrama de flujo del algoritmo de control implementado en LabVIEW Fuente: Elaboración Propia 
Inicialmente se configura la entrada con la temperatura deseada dentro de la cámara de medición. Posteriormente se captura el dato de temperatura actual para realizar el cálculo del error definido como la comparación de la entrada con la salida del lazo de control. El siguiente paso es realizar la integración del error. Enseguida, se calcula el valor de la derivada del error. Para calcular el control proporcional se realiza el producto de la ganancia proporcional por el error. Para poder obtener la salida del controlador, se realiza la suma de los valores previamente calculados. Finalmente se envía el dato de salida al microcontrolador para generar la señal PWM con el ancho de pulso correspondiente a la generación de temperatura de la celda Peltier y esperar por la siguiente muestra.

Es importante mencionar que el sistema tiene la capacidad de manipular la salida del controlador de forma manual, de tal manera que poder realizar pruebas en lazo abierto del mismo en caso de ser necesario. En la figura 6 se muestra la interfaz del software desarrollado aplicando el algoritmo de control propuesto.

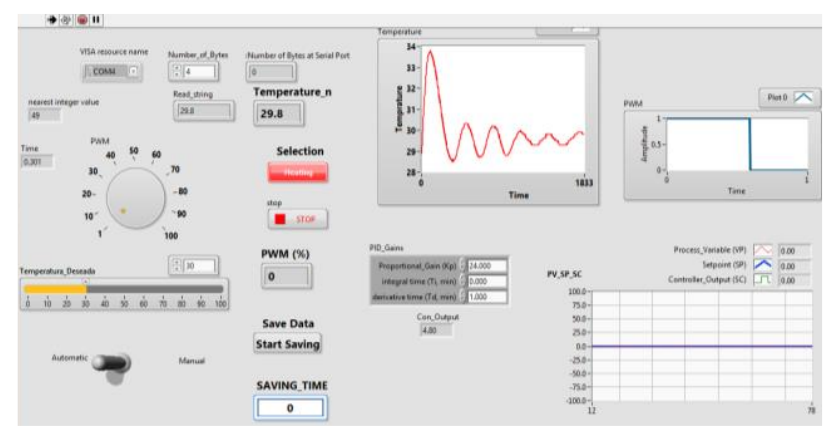

Figura 6 Interfaz desarrollada en LabVIEW Fuente: Elaboración Propia

\section{Resultados Experimentales}

Una vez terminado el diseño de la cámara de medición, se realizó el proceso de manufactura para posteriormente realizar el ensamble final, como se muestra en la figura 7 .
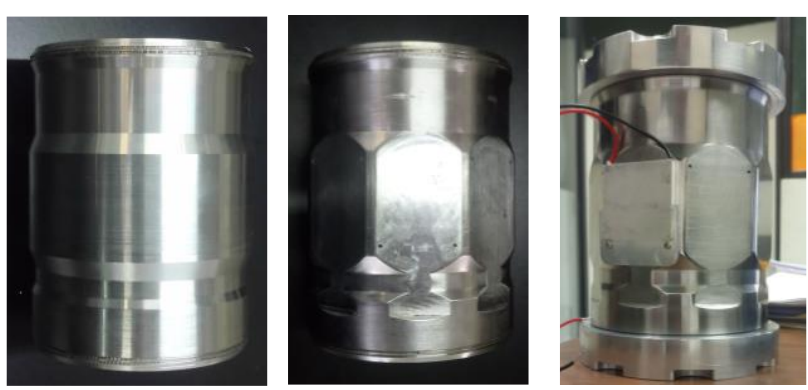

Figura 7 Ensamble de la cámara de medición desarrollada Fuente: Elaboración Propia
Se procedió integrar un arreglo experimental para la medición de la respuesta de los sensores. Se utilizó un sensor de gas óxidometálico semiconductor comercial MQ-3 de la marca Zhengzhou Winsen Electronics Technology Co (Zhengzhou Winsen Electronics Technology Co 2014), el cual tiene una alta sensibilidad a diferentes alcoholes. Por otro lado, se utilizó un sensor de gas QCM de $12 \mathrm{MHz}$ (corte AT) con una película sensible de etíl celulosa con un grosor de aproximadamente 0.7 $\mu \mathrm{m}$. Para realizar las mediciones se aplicaron muestras de etanol a diferentes concentraciones. En la Figura 8 se muestra el arreglo experimental utilizado para el sistema de medición, así como el sistema que mide la respuesta de los sensores utilizados.

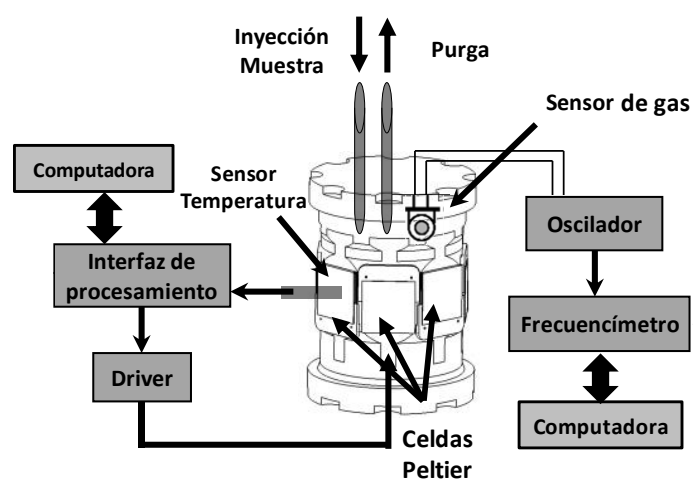

Figura 8 Arreglo experimental utilizado para el control de la cámara y la medición de respuesta de sensores de gas. Fuente: Elaboración Propia

Se realizaron mediciones utilizando un sensor óxido-metálico, aplicando muestras de etanol a diferentes concentraciones. Se realizó la sintonización de ganancias para realizar la regulación de la temperatura con una $\mathrm{Kp}=21$, $\tau_{\mathrm{d}}=1$ y una $\tau_{\mathrm{i}}=0.001$. En este caso, se ajustó la temperatura de la cámara de medición a diferentes temperaturas para observar el comportamiento del sensor. Además, para este arreglo experimental se considera la humedad ambiente alrededor de $35 \%$ de humedad relativa.

El proceso de medición es el siguiente; se inicia el sistema de medición y se ajusta a una temperatura determinada. En paralelo, se inicia el sistema que registra la respuesta del sensor. Una vez que el sistema se ajusta a la temperatura deseada, se espera a que la respuesta del sensor se estabilice en lo que se conoce como línea base. Al estabilizarse la respuesta del sensor se procede a la aplicación de las muestras, en este caso se utilizó etanol a diferentes concentraciones. 
El Gráfico 1, muestra la respuesta del sensor MQ-3 expuesto a una temperatura dentro de la cámara de $20^{\circ} \mathrm{C}$, donde se puede apreciar que el sensor con aire limpio mantiene un offset de aproximadamente $0.3 \mathrm{~V}$. Al aplicar la muestra de 2700 ppm de etanol, muestra una amplitud en el voltaje de salida del sensor de aproximadamente $2.8 \mathrm{~V}$.

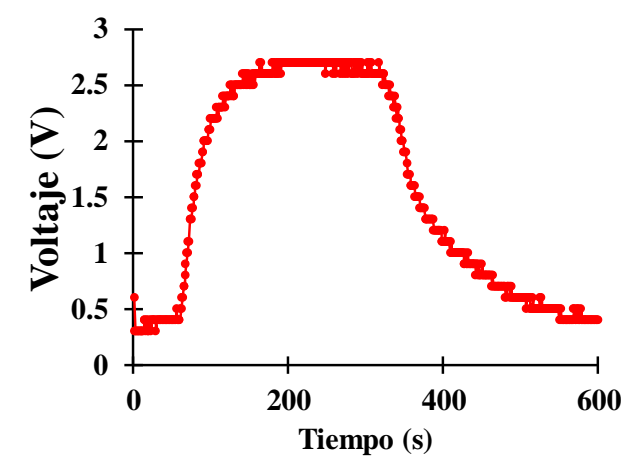

Gráfico 1 Respuesta del sensor de gas MQ-3 a $20{ }^{\circ} \mathrm{C}$ de temperature

Fuente: Elaboración Propia

En el Gráfico 2, se observa la respuesta del sensor MQ-3 con una temperatura de la cámara ajustada a $30^{\circ} \mathrm{C}$, aplicando una muestra de 2700 ppm de etanol. Se puede observar la respuesta del sensor con una amplitud en estado estable en el voltaje de salida de aproximadamente $2.5 \mathrm{~V}$. Al tener un incremento en la temperatura se muestra un decremento en la señal de salida del mismo.

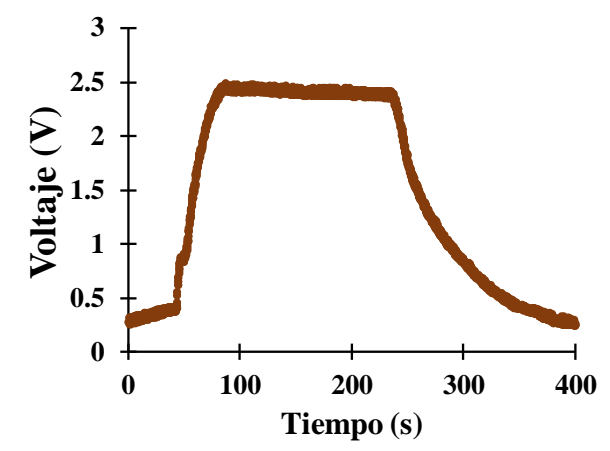

Gráfico 2 Respuesta del sensor de gas MQ-3 a $30{ }^{\circ} \mathrm{C}$ de temperature

Fuente: Elaboración Propia

Para el caso la medición de la respuesta del sensor expuesto a una temperatura de $40{ }^{\circ} \mathrm{C}$, se obtuvo una respuesta con una amplitud en estado estable de $2.3 \mathrm{~V}$, lo que corrobora el hecho de que a una mayor temperatura el sensor disminuye su respuesta, como se muestra en el Gráfico 3. Con estos resultados se puede decir que el sistema funciona de manera adecuada.

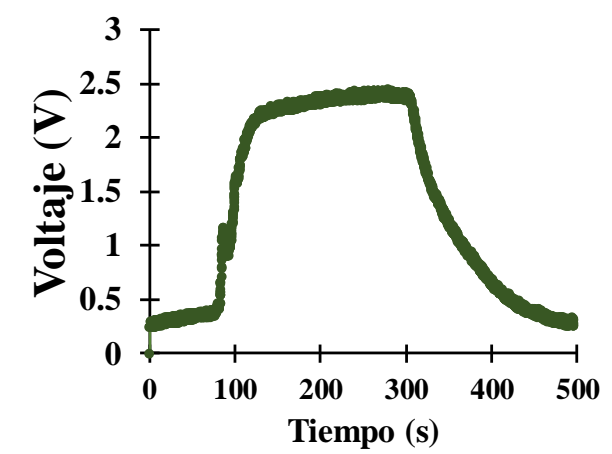

Gráfico 3 Respuesta del sensor de gas MQ-3 a $40{ }^{\circ} \mathrm{C}$ de temperature

Fuente: Elaboración Propia

Finalmente, se realizaron mediciones con el sensor QCM expuesto a etanol ajustando la cámara de medición a varias temperaturas. En este caso, el procedimiento se realizó aplicando tres muestras de $15500 \mathrm{ppm}$ de etanol para cada exposición de temperatura.

En el Gráfico 4 se muestran las respuestas crudas del sensor QCM expuesto a diferentes temperaturas. Se puede apreciar la frecuencia a la cual oscila el sensor, así como los corrimientos de frecuencia en la respuesta del mismo generados para temperaturas en los rangos de 20 ${ }^{\circ} \mathrm{C}, 30{ }^{\circ} \mathrm{C}$ y $40{ }^{\circ} \mathrm{C}$. Se observan los cambios de las líneas base de los sensores debido a las diferencias en las magnitudes de las respuestas generadas al estar expuestos a diferentes temperaturas. Es decir, al ser altamente sensibles a la temperatura, estos sensores muestran un cambio significativo en la frecuencia de resonancia.

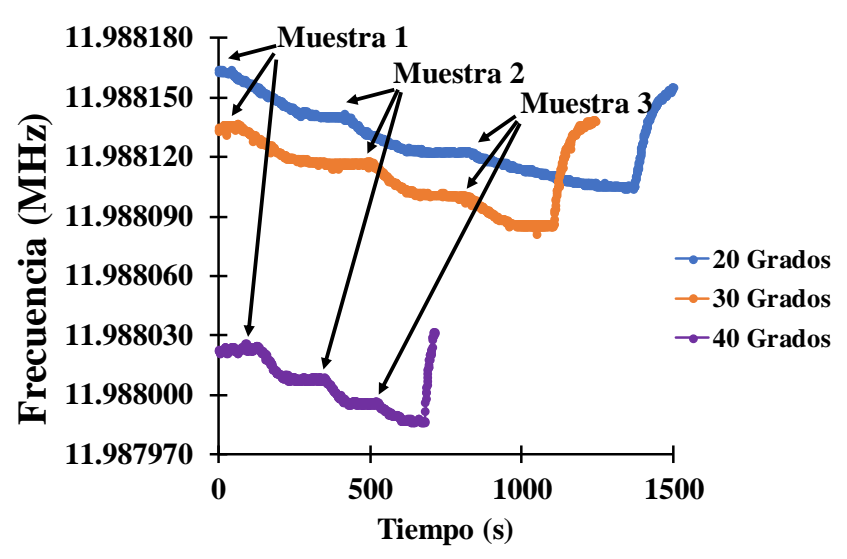

Gráfico 4 Respuestas crudas del sensor QCM expuesto a temperaturas de 20,30 y $40{ }^{\circ} \mathrm{C}$ de temperature Fuente: Elaboración Propia

En Gráfico 5, se muestra (a diferencia de la figura anterior) el decremento de frecuencia $(\Delta \mathrm{f})$ en función de la línea base respecto a cada exposición de temperatura. 
Se puede observar que, al estar el sensor expuesto a una temperatura de $20^{\circ} \mathrm{C}$. Al aplicar la primera muestra de etanol se alcanzó un decremento con una magnitud de $24 \mathrm{~Hz}$. $\mathrm{Al}$ aplicar la segunda muestra, se obtuvo un decremento de $41 \mathrm{~Hz}$. Al aplicar la tercera muestra de etanol, se obtuvo un decremento de $59 \mathrm{~Hz}$ y finalmente purgar la cámara de medición. El tiempo de medición de la respuesta del sensor fue de aproximadamente $1500 \mathrm{~s}$.

Para la respuesta del sensor expuesto a una temperatura de $30{ }^{\circ} \mathrm{C}$. Se obtuvo un decremento con una magnitud de $18 \mathrm{~Hz}$ al aplicar la primera muestra. Al aplicar la segunda muestra de etanol, se observa un decremento de aproximadamente $34 \mathrm{~Hz}$. Al aplicar la tercera muestra se observa un decremento de $49 \mathrm{~Hz}$. El tiempo de medición de respuesta fue de aproximadamente de $1200 \mathrm{~s}$. Finalmente, se expuso el sensor QCM a una temperatura de $40{ }^{\circ} \mathrm{C}$. Al aplicar la primera muestra se observa un decremento en la frecuencia de resonancia de $14 \mathrm{~Hz}$. Aplicar la segunda muestra se puede apreciar un decremento en la magnitud de $28 \mathrm{~Hz}$.

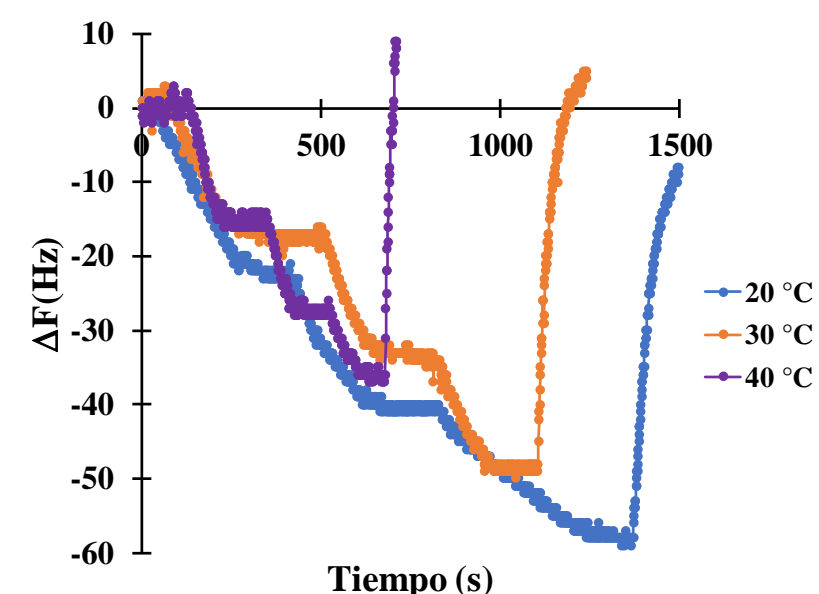

Gráfico 5 Respuesta del sensor de gas $\mathrm{MQ}-3$ a $40^{\circ} \mathrm{C}$ de temperatura

Fuente: Elaboración Propia

Finalmente, al aplicar la tercera muestra se observa un decremento de $37 \mathrm{~Hz}$. El tiempo de medición de respuesta fue de aproximadamente 700 s. Los resultados obtenidos muestran una diferencia en las magnitudes de las respuestas del sensor, así como una diferencia en el tiempo de medición para cada una de las temperaturas a la que fue expuesto el QCM.

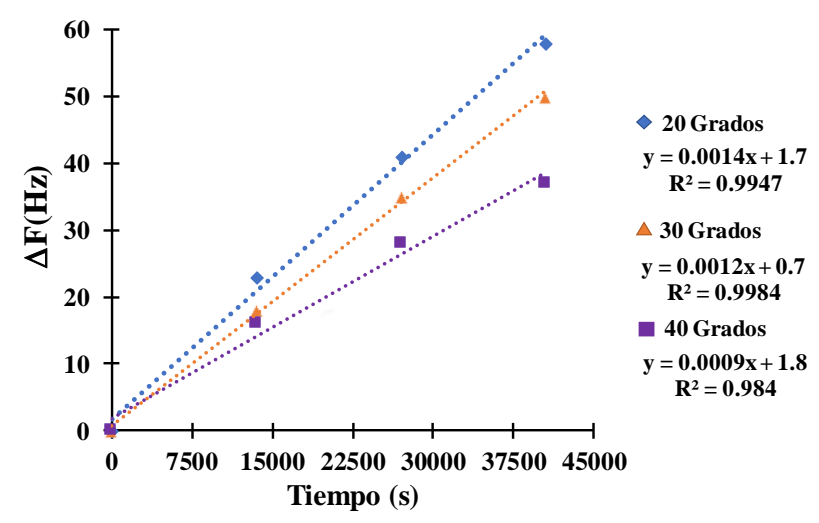

Gráfico 6 Ajuste lineal y coeficiente de correlación Fuente: Elaboración Propia

Finalmente, en el Gráfico 6, se obtuvo la respuesta del sensor para cada temperatura, en función de la concentración para observar la linealidad en el comportamiento del sensor. Se calculó el coeficiente de correlación donde se puede observar un coeficiente de correlación de $\mathrm{R}^{2}=0.9947$ para una temperatura de $20^{\circ} \mathrm{C}$. Para una temperatura de $30{ }^{\circ} \mathrm{C}$ se obtuvo un coeficiente de correlación de $\mathrm{R}^{2}=0.9984$ y para una temperatura de $40{ }^{\circ} \mathrm{C}$, un coeficiente de correlación de $\mathrm{R}^{2}=0.9840$.

Es importante mencionar que el sistema de control de temperatura muestra una estabilidad en la temperatura deseada dentro de la cámara de medición, lo que hace que las respuestas de los sensores de gas sean satisfactorias.

Con los resultados obtenidos tanto de los sensores de óxido metálicos, como para los QCM se ha podido apreciar resultados típicos en la respuesta para este tipo de sensores, con lo que es posible hacer un estudio cuantitativo y cualitativo para poder realizar un estudio profundo de la respuesta de sensores de gas. Por lo tanto, se puede decir que el desarrollo del sistema de medición de sensores de gas funciona de forma correcta, dado que se han obtenido resultados satisfactorios.

\section{Agradecimientos}

Se agradece las facilidades prestadas para desarrollo de este proyecto a la Universidad Tecnológica de Puebla y a la Benemérita Universidad Autónoma de Puebla. 


\section{Conclusiones}

Se ha desarrollado un sistema de medición de respuesta para sensores de gas, en este caso particular para sensores de óxido-metálicos y para sensores QCM. Al realizar las mediciones correspondientes se ha observado un comportamiento típico en la respuesta de dichos sensores, obteniendo resultados altamente satisfactorios. Con el desarrollo de este sistema se pueden obtener resultados puedan aportar sustancialmente un avance científico para el análisis de la respuesta de este tipo de sensores.

El diseño y construcción de la cámara de medición cubre las necesidades necesarias para la medición de repuesta de sensores gas. Se implementó un firmware con un microcontrolador, como interfaz de comunicación entre la cámara de medición y la computadora. Con la interfaz desarrollada se implementó un algoritmo generando un sistema de control Proporcional-Integral-Derivativo (PID) para el ajuste de temperatura requerido y poder realizar la medición de respuesta y caracterización de este tipo de sensores.

\section{Trabajo a Futuro}

Con el desarrollo de este sistema será posible realizar mediciones de sensores de gas a un bajo costo, así como obtener información confiable para poder realizar el análisis cualitativo y cuantitativo de la respuesta de los sensores y poder desarrollar dispositivos para diferentes aplicaciones ya como una nariz electrónica.

Como trabajo a futuro se planea agregar sensor de humedad, así como integrar un sistema único de medición lo suficientemente robusto para poder controlar desde una sola plataforma, el sistema de medición, así como la medición de respuesta de diferentes tipos de sensores.

\section{Referencias}

A. Arnau (2008). Piezoelectric Transducers and Applications, (2da ed.). Berlín, Alemania: Springer.

Abdulrahman Alassi, Mohieddine Benammar, Dan Brett (2017). Quartz Crystal Microbalance Electronic Interfacing Systems: A Review, Sensors, 17, 1-41.
Dallas Semiconductor (2018, 1 septiembre). Programmable Resolution1-Wire Digital Thermometer Data Sheet [Datasheet]. https://datasheets.maximintegrated.com/en/ds/D S18B20.pdf

E. Torrecilla (2013). El gran libro de Catia (1 ${ }^{\mathrm{a}}$ ed.). Barcelona, España: Alfaomega-Marcombo.

G. Sauerbrey et al (1959). Verwendung von Schwingquarzen zur W"agung d"unner Schichten und zur Mikrow"agung Z, Zeitschrift für Physik 155, 206-222.

H. Troy Nagle, R. Gutiérrez-Osuna, S. S. Schiffman (1998). The How and Why of Electronic Noses, IEEE Spectrum, 35, 22-31.

J. W. Gardner, P. N. Bartlett (1994). A brief history of electronic noses, Sensors Actuators B, $18,210-211$.

J. W. Gardner, P. N. Bartlett (1999). Electronic Noses, $\left(1^{\mathrm{a}}\right.$ ed.). New York, USA: Bookcraft.

J. L. Muñoz Mata, et al (2012). Development and implementation of a system to measure the response of quartz crystal resonator-based gas sensor using a field programmable gate array, Measurement Science and Technology, 23(5), 055104.

K. Ogata (1995). Sistemas de Control en Tiempo Discreto ( ${ }^{\mathrm{a}}$ ed.). Edo. de México, México: Prentice Hall.

Lajara Vizcaíno, J., \& Pelegrí Sebastiá, J. (2011). LabVIEW Entorno gráfico de programación $\left(2^{\mathrm{a}}\right.$ ed.). Ciudad de México, México: Alfaomega.

Microchip. (2006, 1 enero). PIC18F2455/2550/ 4455/4550 Data Sheet [Datasheet]. Recuperado 10 octubre, 2017, de https://ww1.microchip.com /downloads/en/devicedoc/39632c.pdf

S. Muñoz, T. Nakamoto et al (2005). Odor Approximation of fruit flavors using a QCM odor sensing system, Sensor an Actuators B, $105,144-149$.

S. Muñoz-Aguirre, T. Nakamoto, et al (2005). Study of deposition of gas sensing films of quartz crystal microbalance using an ultrasonic atomizer, Sensors an Actuators B, 105(2), 144149.

MUÑOZ-MATA, José Lorenzo, ROJAS-GARNICA, Juan Carlos, MUÑOZ-AGUIRRE, Severino y CERVANTES-DE LA ROSA, Juan Pedro. Diseño e implementación de un sistema de medición de respuesta de sensores de gas. Revista de Aplicaciones de la Ingeniería. 2019. 
Zhengzhou Winsen Electronics Technology Co (2014, 1 mayo). Alcohol Gas Sensor Model: MQ-3 Manual,

https://cdn.sparkfun.com/datasheets/Sensors/Bi ometric/MQ-3\%20ver1.3\%20-\%20Manual.pdf.

W. H. King. Jr. (1964). Piezoelectric Sorption Detector, Analytical Chemistry, 36(9), 17351739. 